\title{
Resistance switching in transparent magnetic MgO films
}

O. Jambois $^{\mathrm{a}}$, P. Carreras ${ }^{\mathrm{b}}$, A. Antony ${ }^{\mathrm{b}}$, J. Bertomeu ${ }^{\mathrm{b}}$, C. Martínez-Boubeta ${ }^{\mathrm{a}, *}$

${ }^{\mathrm{a}}$ Dept. Electrònica, MIND-IN2UB, Universitat de Barcelona, Martí i Fanquès 1, 08028 Barcelona, CAT, Spain

b Grup d'Energia Solar, Dept. Física Aplicada i Òptica, Universitat de Barcelona, 08028 Barcelona, Spain

* Corresponding author. Tel.: +34 934039154.

E-mail addresses: cmartinezboubeta@ub.edu,cboubeta@el.ub.es.

\begin{abstract}
We have studied the abrupt and hysteretic changes of resistance in $\mathrm{MgO}$ based capacitor devices. The switching behavior is discussed in terms of the formation and rupture of conduction filaments due to the migration of structural defects in the electric field, together with the redox events which affects the mobile carriers. The results presented in this paper suggest that $\mathrm{MgO}$ transparent films combining ferromagnetism and multilevel switching characteristics might pave the way for a new method for spintronic multibit data storage.
\end{abstract}




\section{Introduction}

The current-induced resistance switching in magnetic tunnel junctions has received a lot of attention due to its potential application in a simplified design for high density non-volatile memories. Various physical effects have been discussed in this regard to explain the induced resistance switching. Spin torque, for instance, could be of a particular interest for the development of multilevel memory cells through the vertical-current-induced domain-wall motion demonstrated both in an amorphous $\mathrm{Al}-\mathrm{O}$ barrier and $\mathrm{MgO}$ crystalline junctions $[1,2]$.

There is another class of devices, usually made in a two terminal metal/insulator/metal structure, which shows voltage-controlled resistance switching [3,4]. Among the various insulators that have been reported thus far, transition metal (sub) oxides such as $\mathrm{CuO}, \mathrm{NiO}, \mathrm{TaO}_{2}, \mathrm{TiO}_{2}, \mathrm{NbO}_{2}$ and $\mathrm{HfO}_{2}$ have attracted the most attention. Different switching modes have been proposed, including localized traps at the metal/oxide interface [5], ion-migration effects coupled to redox processes [6], and the formation of metallic nanoscopic filaments between the two electrodes [7], although the exact process is still unclear. However, there is a general consensus in the literature that the conducting channels are selectively formed at crystalline defects such as grain boundaries and vacancies [8-10]. It is noteworthy that structural defects such as cation vacancies (or first-row element impurities such as $\mathrm{C}$ and $\mathrm{N}$ ) can also become prime candidates for sources of ferromagnetism in wide-gap oxides, exemplified by $\mathrm{CaO}, \mathrm{ZnO}$, and $\mathrm{HfO}_{2}$ [11]. Therefore, a material combining reversible resistive transitions and robust ferromagnetism is expected to provide a new approach to spintronic memristive devices [12]. MgO, which holds importance in many industrial applications, is very attractive due to its matured process technology. On the one hand, $\mathrm{MgO}$ addition to $\mathrm{ZnO}$ layers $(\mathrm{Mg} x$ $\mathrm{Zn}_{1-x} \mathrm{O}$ ) has shown to improve data retention characteristics in non-volatile memory architectures [10]. Moreover, electrical switching that has been observed in $\mathrm{MgO}$ films [13] and $\mathrm{MgO}$-based magnetic tunnel junctions [14- 
16] is attributed either to nanostructural rearrangements of metallic ions in the electrode/barrier interfaces or reversible displacement of oxygen vacancies. On the other hand, defect-induced ferromagnetism in $\mathrm{MgO}$ has already been observed at room temperature [17-21]. Our previous results demonstrate that the presence of cation vacancies has a strong impact on the electronic properties of $\mathrm{MgO}$. The directionality of the oxygen $\mathrm{p}$ orbitals produces a ferromagnetic state and probably influences its chemical reactivity. We expect the holes that are feebly localized on $\mathrm{Mg}$ vacancies could be aligned by an electric field, and thus can modify the magnetic coupling and conductivity. The purpose of this paper is to address evidences for resistive switching in magnetic $\mathrm{MgO}$ thin films with lattice defects situated at the cation sites.

\section{Device preparation and characterization}

Heterostructures were deposited by RF sputtering on $\mathrm{Nb}$ doped $(0.5 \mathrm{wt} \%)$ $\mathrm{SrTiO}_{3}(001)$ single crystal substrate (Crystec). An $80 \mathrm{~nm} \mathrm{MgO}$ layer was first deposited at $450^{\circ} \mathrm{C}$ in an $\mathrm{Ar}-\mathrm{O}_{2}$ mixture. This temperature is close to the optimal annealing temperatures in usual MgO-based tunnel junctions [22]. Growth conditions have been previously observed to control the density of oxygen vacancies within the $\mathrm{MgO}$ [23]. In particular, adding $\mathrm{O} 2$ to the $\mathrm{Ar}$ plasma during $\mathrm{MgO}$ growth also helps in altering the oxygen population and the barrier performance [24]. It is also worth mentioning that these $\mathrm{MgO}$ films show a remarkable structural distortion [25], which is taken as an indication of the existence of cation vacancies in our samples [17]. We surmise $\mathrm{Mg}$ re-evaporates from the substrate faster than it reacts with $\mathrm{O}$ to form $\mathrm{MgO}$ on the substrate so that cation vacancies are stabilized, thus resulting in a transparent magnetic film (the transmittance is above $80 \%$ in the visible spectrum with saturation magnetization values about $10 \mathrm{emu} / \mathrm{cm}^{3}$ ) as reported elsewhere $[17,18,20]$. The sample was then capped with a $100 \mathrm{~nm}$ thick transparent conductive indium-tin-oxide (ITO) layer deposited at room temperature through a shadow mask, defining an area of $1 \mathrm{~mm}$ in diameter. 
The deposition was carried out using an ITO (In2 O3 doped with 10 wt\% $\mathrm{SnO}_{2}$ ) target with 1 mTorr Ar pressure [26]. The crystallinity and the relative high electrical resistivity of those as-deposited ITO films were improved after vacuum annealing at temperatures above $350{ }^{\circ} \mathrm{C}$ for $1 \mathrm{~h}$. However, it should be noted that, the device heterostructure design has, by no means, been optimized for superior performance. Electrical characterization was performed by an Agilent B1500A parameter analyzer in DC sweep mode. All the measurements were performed by the two probe method at room temperature. Measurement convention was to apply the electric potential to the top electrode while the bottom electrode was grounded.

\section{Results and discussion}

The all-oxide STO-n/MgO/ITO-n heterostructure is charming since the oxygen permeability of the electrodes has been demonstrated to affect the resistive switching significantly [27]. At the same time, the asymmetry of the two interfaces should define a stable switching polarity [4]. All these reasons suggest a different result pattern compared to that of Huang et al. [13].

A highly rectifying $\mathrm{I}-\mathrm{V}$ curve (i.e., current can pass in one direction but not in the opposite direction) was observed in the virgin state (Fig. 1). In addition, the $\mathrm{I}-\mathrm{V}$ curves exhibit a hysteretic behavior similar to the one previously reported in $\mathrm{MgO}$-based magnetic tunnel junctions $[15,16]$. The results indicate that the charge carriers in the $\mathrm{MgO}$ are positive, and hence they must be holes [28]. Both the rectifying direction and subsequent switching polarity support a Schottky-like $\mathrm{MgO} / \mathrm{ITO}$ contact and a more electrically conductive bottom STO/MgO interface [29]. The electroforming process by applying a high electric field across the junction (Fig. 1(b)) creates conductance channels of low resistance which are believed to be the sites for the subsequent switching. It is also worth mentioning that visible electroluminescence (photograph in Fig. 1) develops simultaneously with the 
resistance switching [3,30]. Although not properly understood at this time, the close correlation between this luminescence and the memristor behavior probably indicates that both have the very same origin, i.e., the hole-doping induced by the cation vacancies. In this regard, we have shown recently that luminescence emerges at $2.6 \mathrm{eV}$ in similar defective $\mathrm{MgO}$ films [20]. Obviously further work is required before firm conclusions can be drawn.

Repeatable switching cycles were observed after a forming step. The result from Fig. 2 is that the low resistance state changed to an insulating state at a critical voltage (points 3-4 within the figure). When the voltage was swept again, the device changed back to a conducting state. The occurrence of such a bipolar resistive switching within the $\mathrm{MgO}$ layer was confirmed by performing the $\mathrm{I}-\mathrm{V}$ measurement for the $\mathrm{Nb}-\mathrm{STO}$ substrate or ITO electrode alone, where no reversible switching was observed [31]. Furthermore, the I-V curves are quite symmetric for both polarities, which suggest that the current is limited by the bulk active layer, and not by the electrodes. The electric field, given by the voltage divided by the active layer thickness, amounts about $10^{6} \mathrm{~V} / \mathrm{cm}$ which compares well with the values reported in the literature [32]. The voltage for the forming step should depend significantly on the oxide layer thickness, which indicates that significant margin would be available for decreasing the working bias by decreasing the $\mathrm{MgO}$ thickness.

Next, we studied the effects of applying an electric field repeatedly across the film while the current compliance limit was gradually increased. It has been observed in numerous resistive switching devices that memristor performance depends on the sweep frequency and maximum applied voltage/current limit imposed during the measurement [33]. As can be seen from Fig. 3(a), there are variations in the resistance values from one switching cycle to the next. The pulse with higher current leads to larger changes in resistance from the original state, which is a characteristic of multilevel-switch. However, it is a process poorly controlled in our case due to the limitations of the testing apparatus used for the setting of the compliance current, instead of, for instance, a transistor in parallel with a diode $[27,33]$. The new current-voltage 
characteristic is essentially permanent provided the voltage does not exceed the forming voltage. The 10-fold curves shown in Fig. 3(b) illustrate the reproducible nature of the switching process.

\section{Conclusions}

In conclusion, magnetic devices exhibiting a bipolar resistance switching have been fabricated by sandwiching the $\mathrm{MgO}$ films between $\mathrm{n}$-type conducting ITO and STO electrodes. The rectifying $\mathrm{I}-\mathrm{V}$ characteristics of the device in its virgin state suggest the formation of a depletion $\mathrm{p}-\mathrm{n}$ region at the top interface, as resulting from the introduction of oxygen vacancies within the ITO electrode during the annealing process. Holes near the $\mathrm{p}-\mathrm{n}$ interface would diffuse into the n-type region. Thus, a single irreversible forming step at positive voltages prepares the sample for repeatable switching cycles. A possible mechanism for the resistance change is proposed based on the formation and rupture of conduction filaments due to the migration of structural defects in the electric field [4]. Following the fact that, if the hole density is sufficiently high the holes become delocalized [12,34], we propose this type of defect is most likely due to cation vacancies. Reference should also be made to the memristive switching measurements by Nagashima et al. [32] which highlight the role of redox events in oxides with p-type conduction paths. They propose hole carriers, and hence the device conductance, are compensated (enhanced) by a reduction (oxidation) process. In this regard, we had also demonstrated previously that cation vacancies are compensated by hydrogen reduction, thus controlling the reversible switching phenomena of ferromagnetism in these highly defective $\mathrm{MgO}$ films [18]. The agreement between these two types of experiments strengthens the interpretation of the ferromagnetic ordering in $\mathrm{MgO}$ on the basis of the $\mathrm{Mg}$ vacancies which polarize the $\mathrm{O} 2 \mathrm{p}$-dominated uppervalence bands. 


\section{Acknowledgments}

We would like to thank Ll. Balcells for kindly providing us with the STO substrates. C.M. Boubeta grants financial support through the Spanish Ramón y Cajal program.

\section{References}

[1] C. Martinez-Boubeta, J. Appl. Phys. 102 (2007) 043905.

[2] A. Chanthbouala, R. Matsumoto, J. Grollier, V. Cros, A. Anane, A. Fert, A.V. Khvalkovskiy, K.A. Zvezdin, K. Nishimura, Y. Nagamine, H. Maehara, K. Tsunekawa, A. Fukushima, S. Yuasa, Nature Phys. 7 (2011) 1.

[3] G. Dearnaley, A.M. Stoneham, D.V. Morgan, Rep. Progr. Phys. 33 (1970) 1129. [4] For a recent review, please see R. Waser, R. Dittman, G. Staikov, K. Szot, Adv. Mater. 21 (2009) 2632.

[5] X.G. Chen, X.B. Ma, Y.B. Yang, L.P. Chen, G.C. Xiong, G.J. Lian, Y.C. Yang, J.B. Yang, Appl. Phys. Lett. 98 (2011) 122102.

[6] R. Waser, A. Aono, Nature Mater. 6 (2007) 833.

[7] D.S. Jeong, B.J. Choi, C.S. Hwang, J. Appl. Phys. 100 (2006) 113724.

[8] J.J. Yang, M.-X. Zhang, J.P. Strachan, F. Miao, M.D. Pickett, R.D. Kelley, G. Medeiros-Ribeiro, R.S. Williams, Appl. Phys. Lett. 97 (2010) 232102.

[9] T. Fujii, M. Arita, K. Hamada, H. Kondo, H. Kaji, Y. Takahashi, M. Moniwa, I. Fujiwara, T. Yamaguchi, M. Aoki, Y. Maeno, T. Kobayashi, M. Yoshimaru, J. Appl. Phys. 109 (2011) 053702.

[10] X. Cao, X. Li, X. Gao, X. Liu, C. Yang, L. Chen, J. Phys. D: Appl. Phys. 44 (2011) 015302.

[11] For a review, please see A. Zunger, S. Lany, H. Raebiger, Physics 3 (2010) 53.

[12] U. Wurstbauer, C. Śliwa, D. Weiss, T. Dietl, W. Wegscheider, Nature Phys. 6 (2010) 955.

[13] H.-H. Huang, W.-C. Shih, C.-H. Lai, Appl. Phys. Lett. 96 (2010) 193505. 
[14] D. Halley, H. Majjad, M. Bowen, N. Najjari, Y. Henry, C. Ulhaq-Bouillet, W. Weber, G. Bertoni, J. Verbeeck, G. Van Tendeloo, Appl. Phys. Lett. 92 (2008) 212115.

[15] P. Krzysteczko, G. Reiss, A. Thomas, Appl. Phys. Lett. 95 (2009) 112508.

[16] N. Najjari, D. Halley, M. Bowen, H. Majjad, Y. Henry, B. Doudin, Phys. Rev. B 81 (2010) 174425.

[17] C. Martínez-Boubeta, J.I. Beltrán, L1. Balcells, Z. Konstantinović, S. Valencia, D. Schmitz, J. Arbiol, S. Estrade, J. Cornil, B. Martínez, Phys. Rev. B 82 (2010) 024405.

[18] Ll. Balcells, J.I. Beltrán, C. Martínez-Boubeta, Z. Konstantinović, J. Arbiol, Martínez, Appl. Phys. Lett. 97 (2010) 252503.

[19] C. Moyses Araujo, M. Kapilashrami, X. Jun, O.D. Jayakumar, S. Nagar, Y. Wu, Århammar, B. Johansson, L. Belova, R. Ahuja, G.A. Gehring, K.V. Rao, Appl. Phys. Lett. 96 (2010) 232505.

[20] C. Martínez-Boubeta, A. Martínez, S. Hernández, P. Pellegrino, A. Antony, J. Bertomeu, L1. Balcells, Z. Konstantinović, B. Martínez, Solid State Commun. 151 (2011) 751 .

[21] B.M. Maoz, E. Tirosh, M.B. Sadan, G. Markovich, Phys. Rev. B 83 (2011) 161201(R).

[22] Y. Liu, A.N. Chiaramonti, D.K. Schreiber, H. Yang, S.S.P. Parkin, O.G. Heinonen, A.K. Petford-Long, Phys. Rev. B 83 (2011) 165413.

[23] Z. Diao, J.F. Feng, H. Kurt, G. Feng, J.M.D. Coey, Appl. Phys. Lett. 96 (2010) 202506.

[24] D.J. Kim, W.S. Choi, F. Schleicher, R.H. Shin, S. Boukari, V. Davesne, C. Kieber, J. Arabski, G. Schmerber, E. Beaurepaire, W. Jo, M. Bowen, Appl. Phys. Lett. 97 (2010) 263502.

[25] C. Martinez-Boubeta, Z. Konstantinović, Ll. Balcells, S. Estradé, J. Arbiol, Cebollada, B. Martínez, Cryst. Growth Des. 10 (2010) 1017.

[26] The resistivity of the as deposited $100 \mathrm{~nm}$ ITO film under these conditions on corning $1737 \mathrm{~F}$ glass substrate was $9 \times 10^{-4} \Omega \mathrm{cm}$. Resistivity for $\mathrm{MgO}$ amounts 
about $10^{10} \Omega \mathrm{cm}$.

[27] L. Goux, P. Czarnecki, Y.Y. Chen, L. Pantisano, X.P. Wang, R. Degraeve, Govoreanu, M. Jurczak, D.J. Wouters, L. Altimime, Appl. Phys. Lett. 97 (2010) 243509.

[28] H.R. Day, Phys. Rev. 91 (1953) 822.

[29] T. Susaki, A. Makishima, H. Hosono, Phys. Rev. B 83 (2011) 115435.

[30] H.-M. Benia, P. Myrach, N. Nilius, New J. Phys. 10 (2008) 013010.

[31] X.T. Zhang, Q.X. Yu, Y.P. Yao, X.G. Li, Appl. Phys. Lett. 97 (2010) 222117.

[32] K. Nagashima, T. Yanagida, K. Oka, M. Knai, A. Klamchuen, J.-S. Kim, B.H. Park, T. Kawai, Nano Lett. 11 (2011) 2114.

[33] A. Chen, Appl. Phys. Lett. 97 (2010) 263505.

[34] K.P. McKenna, A.L. Shluger, Proc. R. Soc. A 467 (2011) 1880. 
Fig. 1. The $\mathrm{I}-\mathrm{V}$ characteristics for $\mathrm{STO} / \mathrm{MgO} / \mathrm{ITO} \mathrm{n} / \mathrm{p} / \mathrm{n}$ structure. (a) The initial measurements exhibit rectifying characteristics. The dc voltage was swept from negative to positive values and back, as shown by the arrows. Inset illustrates the device geometry. Following an electroforming step, (b) the device was switched on (showing an abrupt change in conductivity) by applying a positive bias. The current increase was limited by a compliance of $4 \mathrm{~mA}$. Please note the change in vertical axes scale. Inset shows luminescence from a magnetic sample. The luminescence experiments were performed in dark, though the sample was partially illuminated during the photographic exposure.
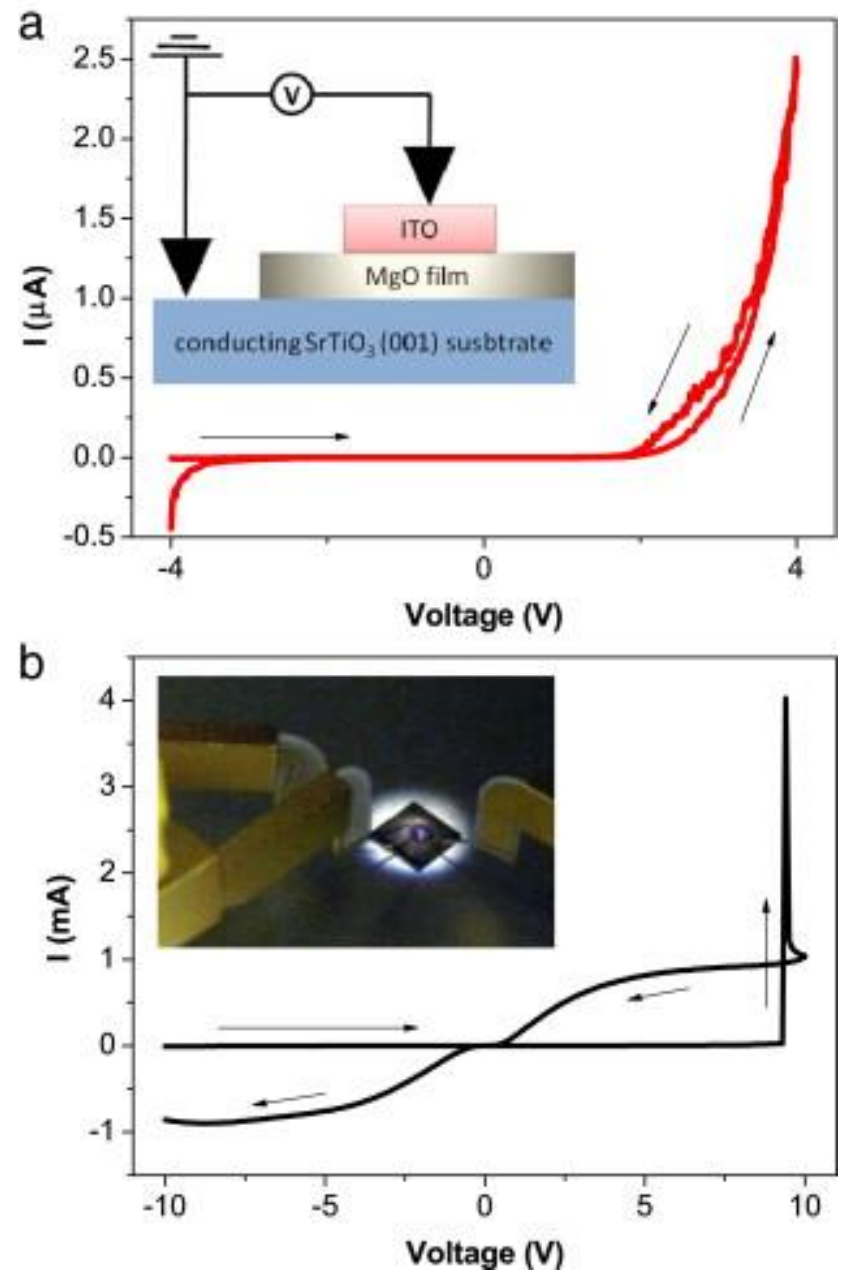
Fig. 2. Reversible bipolar switching $\mathrm{I}-\mathrm{V}$ (a), and resistance loop (b) for the MgO-based device exhibiting two resistive states: a low resistance state, also referred to as the ON state and a high resistance state, referred to as the OFF state. ON/OFF conductance ratio remains about 100. Numbers $1-6$ indicate the bias sweep direction.
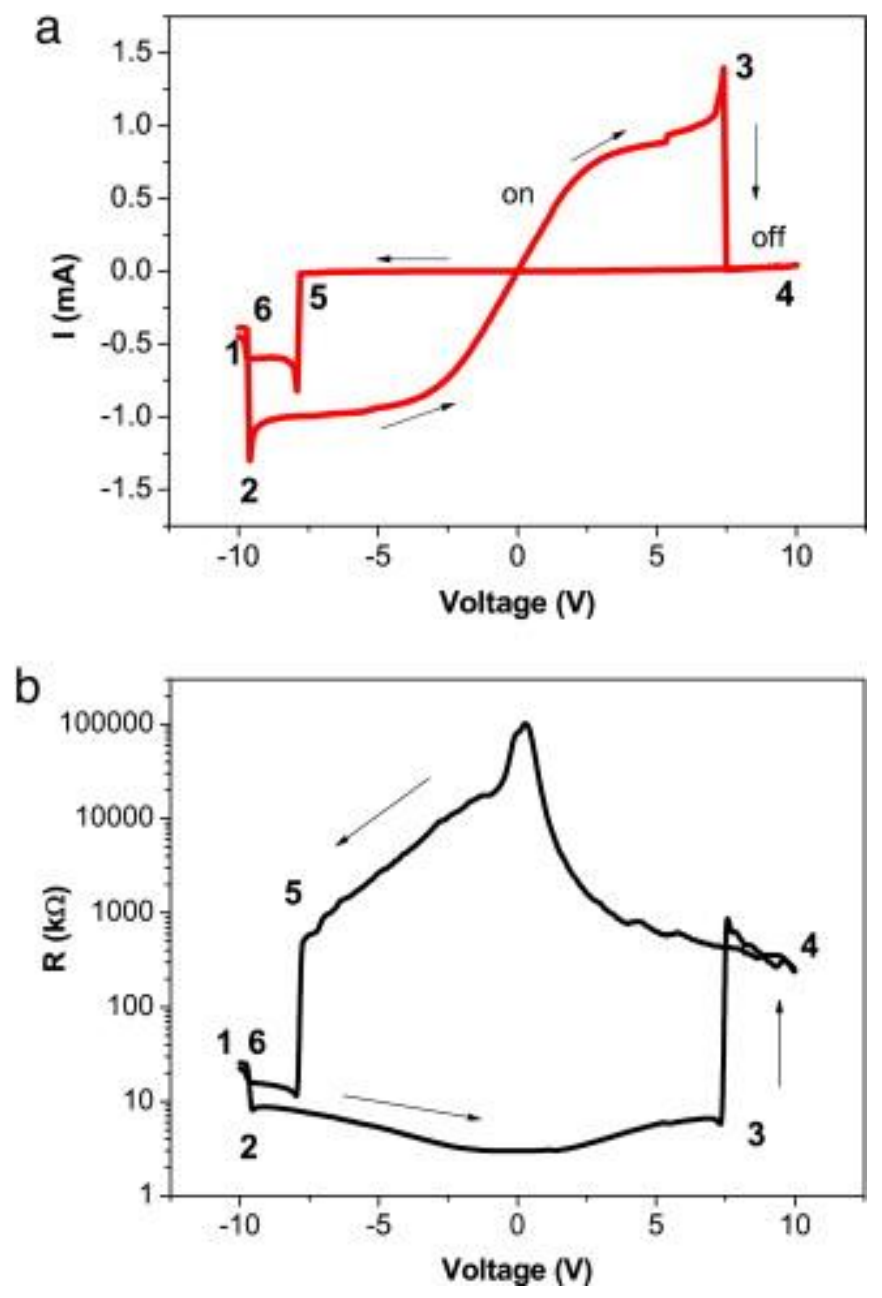
Fig. 3. (a) Multilevel resistance switching traces achieved by setting a compliance current of $4 \mathrm{~mA}$ (continuous heavy line) or $7 \mathrm{~mA}$ (open squares) during positive electroforming process. (b) Endurance test in the $\pm 10 \mathrm{~V}$ bias region: three consecutive sweeps are shown. Minor loop denotes the retention property of the high resistance (OFF) state at moderate voltages. No obvious degradation is observed after the 10th switching loop. Inset: typical semilog I-V characteristics.
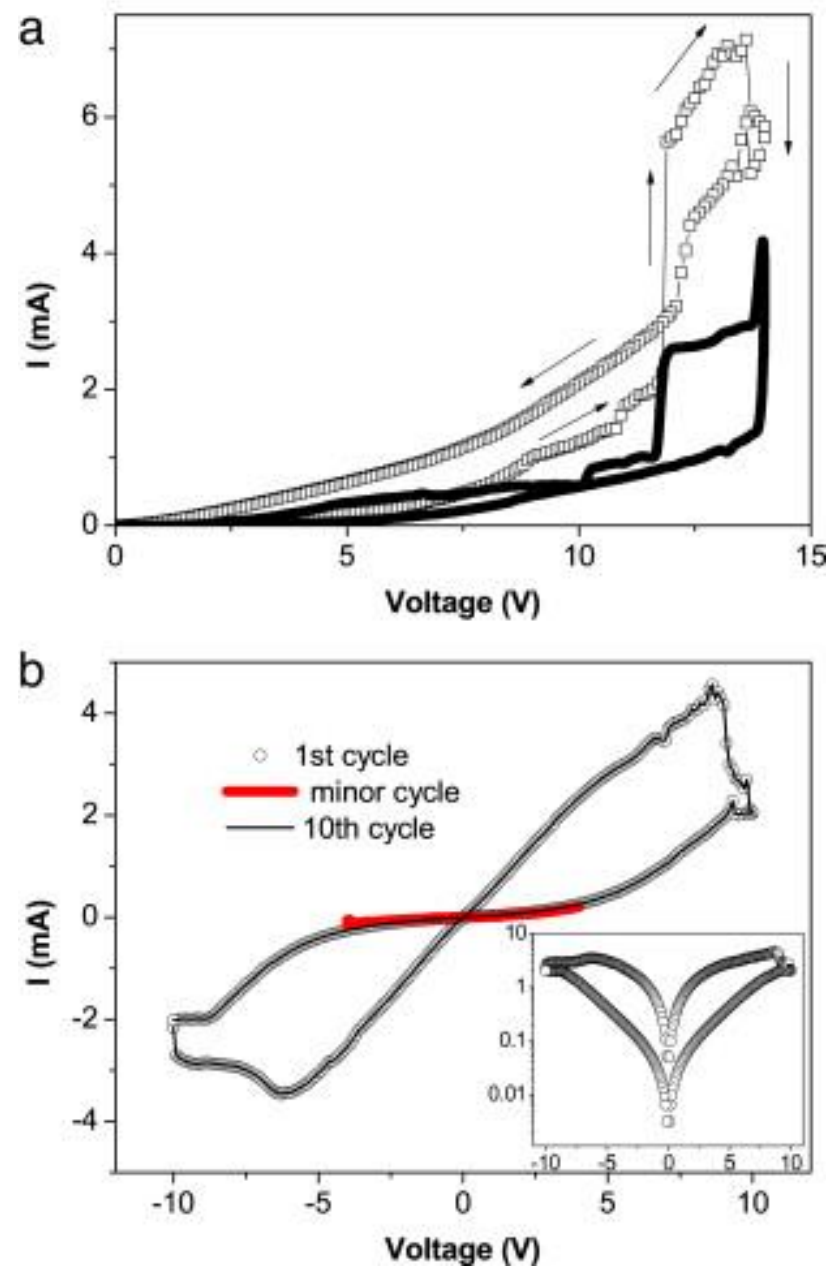